\title{
PROTEIN KINASE C ACTIVATION BEFORE CARDIOPLEGIC ARREST: BENEFICIAL EFFECTS ON MYOCYTE CONTRACTILITY
}

Seung-Jun $\mathrm{O}, \mathrm{MD}$

Monty H. Cox, BS

Fred A. Crawford, Jr., MD

Francis G. Spinale, MD, PhD
Objective: A potential intracellular mechanism for the protective effects of myocardial preconditioning is the activation of protein kinase $\mathrm{C}$. The present study tested the hypothesis that a brief period of protein kinase $\mathrm{C}$ activation before cardioplegic arrest would provide protective effects on myocyte contractility with subsequent reperfusion and rewarming. Methods: Left ventricular porcine myocytes were assigned to the following treatments: (1) Protein kinase C/cardioplegia: Protein kinase $\mathrm{C}$ activation in myocytes $(n=39)$ for 3 minutes with a phorbol ester $\left(10^{-9} \mathrm{~mol} / \mathrm{L}\right.$ of phorbol 12-myristate 13-acetate) in oxygenated, normothermic $\left(37^{\circ} \mathrm{C}\right)$ cell media. Protein kinase $\mathrm{C}$ activation was followed by 2 hours of cardioplegic arrest $\left(\mathrm{K}^{+}, 24 \mathrm{mEq} / \mathrm{L} ; \mathrm{HCO}_{3}{ }^{-}, 30 \mathrm{mEq} / \mathrm{L} ; 4^{\circ} \mathrm{C}\right)$ and a 5 -minute reperfusion period (37 ${ }^{\circ} \mathrm{C}$ media). (2) Cardioplegia: Myocytes $(n=31), 2$ hours of cardioplegic arrest, and a 5-minute reperfusion and rewarming period. Myocyte contractility was measured by means of high-speed videomicroscopy. For comparison purposes, contractile function was examined in myocytes $(n=$ 70) under normothermic control conditions. Results: Myocyte shortening velocity was reduced after cardioplegic arrest when compared with normothermic values $(22.3 \pm 1.6 \mathrm{vs} 48.8 \pm 2.0 \mu \mathrm{m} / \mathrm{sec}, p<0.0001)$. Protein kinase $\mathrm{C}$ activation before cardioplegic arrest normalized myocyte shortening velocity $(48.8 \pm 2.5 \mu \mathrm{m} / \mathrm{sec})$. Co-incubation with phorbol 12-myristate 13-acetate and chelerythrine $\left(10^{-6} \mathrm{~mol} / \mathrm{L}\right)$, an inhibitor of protein kinase $C$, before cardioplegic arrest abolished the protective effects of phorbol 12-myristate 13-acetate pretreatment. Conclusion: These results suggest that an endogenous means of providing improved myocardial protection during prolonged cardioplegic arrest can be achieved through a brief period of protein kinase C activation. (J Thorac Cardiovasc Surg 1997;114:651-9)
D elivery of a hypothermic, hyperkalemic cardioplegic solution is a common means to obtain myocardial quiescence during cardiac surgical procedures. However, transient left ventricular (LV) dysfunction can occur with subsequent reperfusion and rewarming. Myocardial preconditioning describes a phenomenon in which myocardium made transiently ischemic becomes more tolerant to a subsequent and prolonged period of ischemia. ${ }^{1-3}$ Experimental studies have demonstrated that isch-

From the Division of Cardiothoracic Surgery, Medical University of South Carolina, Charleston, S.C.

Supported by National Institutes of Health grant HL-45024, a Basic Research Grant from Pfizer Inc., a Grant-in-Aid from the South Carolina Heart Association, and a Grant-in-Aid from the American Heart Association. M. H. C. performed this work as a Medical Student Research Fellow of the American Heart Association. F. G. S. is an Established Investigator of the American Heart Association. emic preconditioning before the induction of cardioplegic arrest can improve LV pump function with reperfusion and rewarming. ${ }^{4-6}$ Identification of the intracellular mechanism(s) that invoke the preconditioning phenomenon may provide important information for developing novel strategies for myocardial protection during prolonged periods of cardioplegic arrest. Recent studies have demonstrated that activation of the serine-threonine protein kinase $\mathrm{C}(\mathrm{PKC})$ may be an intracellular triggering

Received for publication August 6, 1996; revisions requested Dec. 5, 1996; revisions received April 21, 1997; accepted for publication April 22, 1997.

Address for reprints: Francis G. Spinale, MD, PhD, Cardiothoracic Surgery, Room 418 CSB, Medical University of South Carolina, 171 Ashley Ave., Charleston, SC 29425.

Copyright (C) 1997 by Mosby-Year Book, Inc.

$0022-5223 / 97 \$ 5.00+0 \quad \mathbf{1 2 / 1 / 8 2 9 2 0}$ 
mechanism for the preconditioning phenomenon. ${ }^{7-13}$ However, whether and to what extent direct activation of PKC at the level of the myocyte provides protective effects in the setting of cardioplegic arrest and rewarming remains unknown. Accordingly, the present study was designed to test the central hypothesis that a short interval of PKC activation before a prolonged period of hypothermic, hyperkalemic cardioplegic arrest would improve myocyte contractile function on reperfusion and rewarming.

Pharmacologic activation of PKC has been demonstrated to occur in the presence of phorbol esters. $^{7,10-13}$ This laboratory has described previously that cardioplegic arrest with subsequent reperfusion and rewarming can be successfully performed in isolated LV myocytes. ${ }^{14-16}$ This isolated myocyte system provides an uncomplicated means to examine cellular and molecular events that occur with respect to myocyte contractile processes during cardioplegic arrest and rewarming. Past reports have demonstrated that preconditioning can be successfully induced in isolated myocyte systems. $12,13,16,17$ A brief exposure of isolated myocytes to phorbol esters has been shown to increase PKC activity. ${ }^{13,18,19}$ Accordingly, the present study used an isolated myocyte system of cardioplegic arrest to determine whether a short pretreatment interval with a phorbol ester and subsequent $\mathrm{PKC}$ activation would provide protective effects on myocyte contractile processes.

\section{Methods}

Rationale. The goal of the present study was to examine whether a brief interval of PKC activation before a prolonged period of simulated cardioplegic arrest would protect myocyte contractile function with rewarming. The first series of experiments was designed to develop a protocol for PKC activation in this myocyte system by examining the time and dose-dependent effects of phorbol ester administration on myocyte contractile function under steady-state normothermic conditions. The next series of experiments examined whether a brief PKC activation period before cardioplegic arrest would provide protective effects on myocyte contractile function with subsequent rewarming. The final series of experiments was performed to determine more carefully that the effects of phorbol ester treatment on myocyte function were due to specific PKC activation.

Myocyte isolation and contractile function. Adult Yorkshire pigs $(n=5)$ were the source of LV myocytes. All animals were treated and cared for in accordance with the National Institutes of Health "Guide for the Care and Use of Laboratory Animals" (National Research Council, Washington, D.C., 1996). The animals were anesthetized with isoflurane $(2 \% / 1.5 \mathrm{~L} / \mathrm{min})$ and their lungs ventilated through a nonrecirculating anesthesia circuit. A sternotomy was then performed, and the heart was quickly extirpated and placed in cold oxygenated Krebs solution. The region of the LV free wall perfused by the left circumflex coronary artery $(5 \times 5 \mathrm{~cm})$ was dissected free, cannulated, and prepared for myocyte isolation as described previously. ${ }^{14-16}$ In brief, oxygenated modified Krebs solution containing aerobic substrates and collagenase $(0.5 \mathrm{mg} / \mathrm{ml}$, type II; $146 \mathrm{U} / \mathrm{mg}$, Worthington Biochemical Corp., Freehold, N.J.) was perfused and recirculated through the cannulated circumflex artery for 20 minutes. The tissue was then minced into $2 \mathrm{~mm}$ sections and added to an oxygenated solution containing bovine serum albumin (2\%, Sigma Chemical Co., St. Louis, Mo.), deoxyribonuclease (DNase, 51 Kunitz units/ml, type IV, Sigma $), \mathrm{CaCl}_{2}\left(400 \times 10^{-6} \mathrm{~mol} / \mathrm{L}\right.$, Sigma $)$, and collagenase $(0.5 \mathrm{mg} / \mathrm{ml}$, Worthington $)$ and gently agitated. The isolated myocytes were then suspended in fresh culture medium (media 199, $2 \mathrm{mmol} / \mathrm{L} \mathrm{Ca}^{2+}$, Gibco Laboratories, Grand Island, N.Y.). A $2 \mathrm{ml}$ aliquot of the isolated myocyte suspension $\left(5 \times 10^{4}\right.$ cells/ml) was then plated onto coverslips previously coated with a laminin/fibronectin matrix (Matrigel, Collaborative Research, Inc., Bedford, Mass.) and incubated at $37^{\circ} \mathrm{C}$ for 1 hour in the presence of $95 \%$ oxygen and $5 \%$ carbon dioxide.

Myocyte contractile function was examined with the use of computer-assisted videomicroscopy techniques described previously. ${ }^{14-16}$ In brief, myocytes were imaged on an inverted microscope (model PIM, World Precision Instruments, Sarasota, Fla.) in a $2.5 \mathrm{ml}$ tissue chamber with a thermoregulator to maintain media temperature at $37^{\circ} \mathrm{C}$. Myocytes were stimulated at $1 \mathrm{~Hz}$ and contractions were imaged with the use of a charge-coupled device (GPCD60, Panasonic, Secaucus, N.J.). Myocyte motion signals were input through an edge-detector system (Crescent Electronics, Sandy, Utah), converted into a voltage signal, digitized, and input into a computer (80286, ZBV2526, Zenith Data Systems, St. Joseph, Mo.) for subsequent analysis. Stimulated myocytes were allowed a 5-minute stabilization period, after which contraction data for each myocyte were recorded for a minimum of 20 consecutive contractions. Parameters computed from the digitized contraction profiles included percent shortening (percent), peak velocity of shortening (micrometers per second), peak velocity of relengthening (micrometers per second), total contraction duration (milliseconds), time to peak contraction (milliseconds), and time to $50 \%$ relaxation (milliseconds).

PKC activation and myocyte contractile function. To develop a protocol for PKC activation in isolated myocytes, we used the initial series of experiments to examine the effects of a phorbol ester, phorbol 12-myristate 13acetate (PMA, Sigma), on myocyte contractile function under normothermic conditions. The phorbol ester PMA was selected for these studies because it has been demonstrated previously to activate PKC in isolated myocyte preparations ${ }^{11}$ and readily enters the intracellular compartment. Stock PMA was solubilized with $100 \%$ ethanol (Pharmco Products. Inc., Brookfield, Conn.) and then diluted with a Krebs buffer solution. Myocyte contractile function and viability were examined before and after incubation ( 3 to 30 minutes) with PMA $\left(10^{-6}\right.$ to $10^{-9}$ $\mathrm{mol} / \mathrm{L}$ ). In the present study, a significant reduction in 
myocyte contractile function occurred at $10^{-9} \mathrm{~mol} / \mathrm{L}$ of PMA with no change in myocyte viability (Fig. 1). However, myocyte viability, as defined in the previous section, was substantially reduced with PMA at concentrations greater than $10^{-8} \mathrm{~mol} / \mathrm{L}$ and for incubation intervals greater than 10 minutes. Accordingly, incubation of myocytes with PMA at a concentration of $10^{-9} \mathrm{~mol} / \mathrm{L}$ for 3 minutes was selected as the pretreatment protocol for the simulated cardioplegia experiments.

\section{Simulated cardioplegic arrest with rewarming}

Effect of PKC activation. The next series of experiments was performed to examine whether a brief period of PMA pretreatment before cardioplegic arrest would provide protective effects on myocyte contractile function on rewarming. Isolated myocytes were randomly assigned to one of the following treatment groups: (1) incubation in oxygenated, normothermic media $\left(37^{\circ} \mathrm{C}\right)$ for 3 minutes; (2) incubation in oxygenated, normothermic media containing PMA $\left(10^{-9} \mathrm{~mol} / \mathrm{L}\right)$ for 3 minutes; or (3) incubation in oxygenated, normothermic media containing vehicle (Krebs buffer solution containing equivalent concentrations of ethanol) for 3 minutes. All myocytes were then subjected to simulated cardioplegic arrest as described previously. ${ }^{14-16}$ Specifically, myocytes were exposed to a crystalloid cardioplegic solution (lactated Ringer's solution: $\mathrm{K}^{\div}, 24 \mathrm{mEq} / \mathrm{L} ; \mathrm{HCO}_{3}^{-}, 30 \mathrm{mEq} / \mathrm{L}$; oxygen tension > $300 \mathrm{~mm} \mathrm{Hg}$ ) for 2 hours at $4^{\circ} \mathrm{C}$. After cardioplegic arrest, the cardioplegic solution was rapidly changed with normothermic cell culture medium. After 5 minutes of normothermic reperfusion, steady-state myocyte contractile function was examined. The experimental protocol for this series of studies is shown in Fig. 2.

Effects of PKC blockade. A series of experiments was performed to examine whether the effects of PMA on myocyte contractile function before cardioplegic arrest with rewarming were specifically mediated by activation of PKC. Chelerythrine (LC Laboratory, Woburn, Mass.) has been demonstrated to be a potent (inhibitory concentration of $\left.50 \%\left[\mathrm{IC}_{50}\right]=10^{-7} \mathrm{~mol} / \mathrm{L}\right)$ and specific inhibitor of the catalytic domain of $\mathrm{PKC} .{ }^{20}$ In a past report, chelerythrine at a concentration of $10^{-6} \mathrm{~mol} / \mathrm{L}$ abolished the protective effects of preconditioning in human atrial tissue preparations. ${ }^{7}$ Accordingly, myocytes were incubated in normothermic, oxygenated media containing both chelerythrine $\left(10^{-6} \mathrm{~mol} / \mathrm{L}\right)$ and PMA $\left(10^{-9} \mathrm{~mol} / \mathrm{L}\right)$ together for 3 minutes, then subjected to identical treatment as described in the previous paragraph. In an additional set of experiments, myocytes were exposed to chelerythrine $\left(10^{-6} \mathrm{~mol} / \mathrm{L}\right)$ for 3 minutes and then subjected to cardioplegic arrest. The experimental protocol for these studies is outlined in Fig. 2.

PKC translocation in isolated myocytes. A generalized intracellular event that occurs after activation of $\mathrm{PKC}$ is translocation from intracellular cytosolic compartments to the cell membrane. ${ }^{21-23}$ Furthermore, it has been demonstrated previously that PMA causes PKC translocation in isolated myocyte preparations. ${ }^{23}$ Accordingly, localization of PKC in isolated myocytes was performed by means of fuorescent techniques. ${ }^{24}$ Isolated myocytes from three different preparations were assigned to one of the following treatment groups: (1) incubation in oxygenated, normothermic media $\left(37^{\circ} \mathrm{C}\right.$ ) for 3 minutes; (2) incubation in oxygenated, normothermic media containing PMA $\left(10^{-9}\right.$
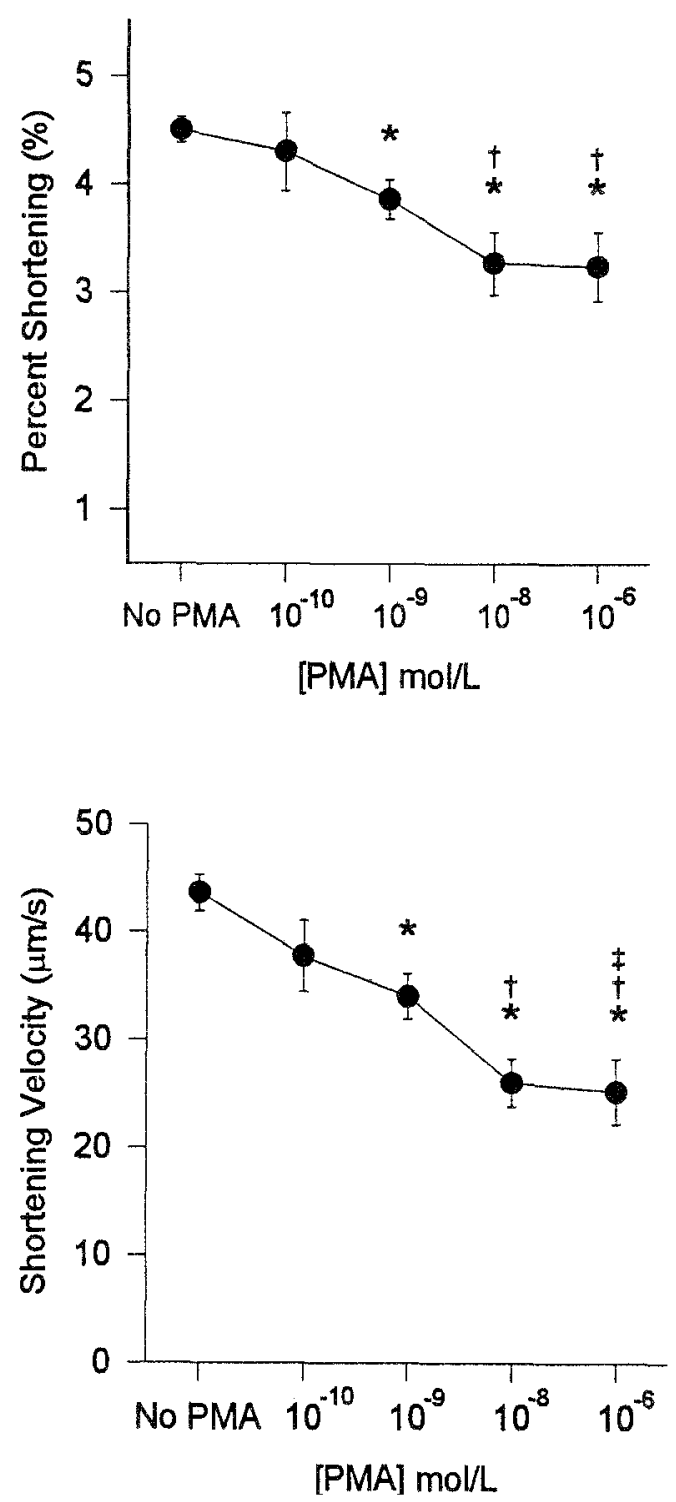

Fig. 1. Effects of PKC activation on myocyte contractile function in the presence of the phorbol ester PMA. The purpose of the first series of experiments was to determine the dose-dependent effect of PMA $\left(10^{-6}\right.$ to $\left.10^{-10} \mathrm{~mol} / \mathrm{L}\right)$ on myocyte contractile function under normothermic conditions. In the presence of PMA, a dose-dependent decline in steady-state myocyte contractile function was observed with a significant reduction occurring at $10^{-9} \mathrm{~mol} / \mathrm{L}$ of PMA. Accordingly, $10^{-9} \mathrm{~mol} / \mathrm{L}$ of PMA was selected as the concentration to be used for PKC activation in isolated myocytes for the cardioplegic arrest and rewarming studies. A minimum number of 15 myocytes were studied at each concentration of PMA. * $p<0.05$ vs no PMA; $\dagger p<0.05$ vs [PMA] $10^{-10} \mathrm{~mol} / \mathrm{L}$; f $<0.05$ vs $[\mathrm{PMA}] 10^{-9} \mathrm{~mol} / \mathrm{L}$ ).

$\mathrm{mol} / \mathrm{L}$ ) for 3 minutes; and (3) incubation in oxygenated, normothermic media containing both chelerythrine $\left(10^{-6}\right.$ $\mathrm{mol} / \mathrm{L})$ and PMA $\left(10^{-9} \mathrm{~mol} / \mathrm{L}\right)$ together for 3 minutes. After these treatments, myocytes were fixed in $3.7 \%$ 


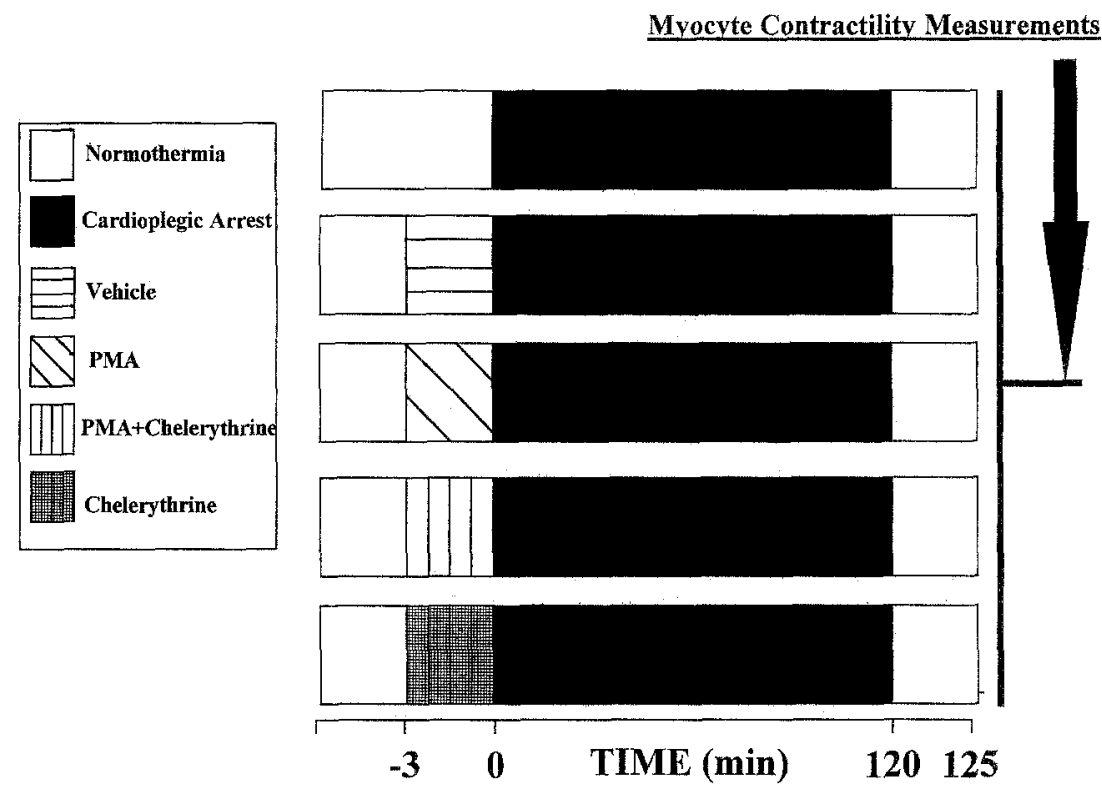

Fig. 2. Schematic representation of myocyte treatment protocols. Normothermia is maintenance of myocytes in oxygenated cell media at $37^{\circ} \mathrm{C}$. Cardioplegic arrest is incubation of myocytes with crystalloid cardioplegic solution $\left(24 \mathrm{mEq} / \mathrm{L} \mathrm{K}^{+}\right)$at $4^{\circ} \mathrm{C}$ for 2 hours. Vehicle is incubation of myocytes in oxygenated normothermic media containing Krebs buffer solution containing ethanol $(0.01 \%)$. PMA is incubation of myocytes in normothermic, oxygenated media containing $10^{-9} \mathrm{~mol} / \mathrm{L}$ of PMA for 3 minutes. $P M A+$ Chelerythrine is incubation of myocytes in normothermic, oxygenated media containing PMA $\left(10^{-9}\right.$ $\mathrm{mol} / \mathrm{L})$ and chelerythrine $\left(10^{-6} \mathrm{~mol} / \mathrm{L}\right)$ for 3 minutes. After the treatment protocols, indices of myocyte contractile function at steady-state were obtained.

molecular grade formaldehyde for 10 minutes and then transferred to a stabilization buffer containing 1,4 Peperazinedithanesulfonic acid (PIPES) $(65 \mathrm{mmol} / \mathrm{L})$, HEPES $(25 \mathrm{mmol} / \mathrm{L})$, ethyleneglycol tetraacetic acid (EGTA) $(10$ $\mathrm{mmol} / \mathrm{L})$, and $\mathrm{MgCl}_{2}(3 \mathrm{mmol} / \mathrm{L})$. The myocytes were then incubated in the presence of a fluorescently conjugated PKC probe (BIODIPY FL bis-indolylmaleimide; 100 $\mu \mathrm{mol} / \mathrm{L}, \mathrm{B} 7485$ Molecular Probes, Eugene, Ore. $)^{24}$ for 10 minutes. Fluorescently conjugated bis-indolylmaleimide has been demonstrated previously to be membrane-permeant, bind to the catalytic domain of $\mathrm{PKC}$, and demonstrate translocation of $\mathrm{PKC}$ from the cytoplasm to membranes. ${ }^{24}$ In preliminary studies, the concentration and incubation period for this $\mathrm{PKC}$ probe provided specific intracellular staining in isolated LV myocyte preparations. After the incubation step, the coverslips containing the myocytes were placed on glass slides containing an antifade glycerol solution (4\%, 1,8 diazabicyclo[2.2.2]octane, Sigma). The myocyte preparations were then examined by means of confocal scanning microscopy (MRC-1000, BioRad Laboratories, Hercules, Calif.) with excitation/emission wavelength settings of $509 / 520$, respectively. Myocytes were imaged at an original magnification of $600 x$ and images digitally captured at $1.0 \mu \mathrm{m}$ increments through the myocyte. A minimum of 25 myocytes were examined from each preparation.

Data analysis. Indices of myocyte contractile function for the different treatment groups were compared by means of analysis of variance. If analysis of variance detected signifi- cant differences with respect to treatment groups, mean separation was performed with the use of Bonferroni bounds. All statistical procedures were performed with the use of the BMDP statistical software package (BMDP Statistical Software, Inc., Los Angeles, Calif.). Results are presented as mean \pm standard error of the mean. Values of $p<0.05$ were considered statistically significant.

\section{Results}

Steady-state myocyte contractile function under normothermic conditions, after simulated cardioplegic arrest with rewarming, and with PMA pretreatment followed by cardioplegic arrest and rewarming are presented in Table I. Indices of contractile function obtained under steady-state normothermic conditions are consistent with previous values obtained with the use of this myocyte preparation. ${ }^{14-16}$ After cardioplegic arrest and rewarming, steadystate myocyte percent and velocity of shortening were reduced by more than $40 \%$ from normothermic control values. The reduction in myocyie contractility after simulated cardioplegic arrest and rewarming is consistent with previous reports from this laboratory. ${ }^{14-16}$ Pretreatment with PMA before cardioplegic arrest and rewarming resulted in improved indices of myocyte contractile function with 
Table I. Steady-state myocyte contractile function after cardioplegic arrest and rewarming: Effects of protein kinase $C$ activation before cardioplegic arrest

\begin{tabular}{|c|c|c|c|}
\hline & Normothermia & Cardioplegia & PMA/cardioplegia \\
\hline Percent shortening $(\%)$ & $4.5 \pm 0.2$ & $2.1 \pm 0.1^{*}<0.0001$ & $4.3 \pm 0.2$ \\
\hline Shortening velocity $(\mu \mathrm{m} / \mathrm{sec})$ & $48.8 \pm 2.0$ & $22.3 \pm 1.6^{*}<0.0001$ & $48.8 \pm 2.5$ \\
\hline Relengthening velocity $(\mu \mathrm{m} / \mathrm{sec})$ & $46.7 \pm 2.2$ & $18.0 \pm 1.5^{*}<0.0001$ & $49.0 \pm 3.5$ \\
\hline Total duration (msec) & $482.5 \pm 9.8$ & $617.7 \pm 36.0^{*}<0.0001$ & $553.15 \pm 21.9^{*} 0.0058$ \\
\hline Time to peak contraction (msec) & $243.6 \pm 7.2$ & $306.8 \pm 16.1 * 0.0002$ & $289.0 \pm 13.7 * 0.0038$ \\
\hline Time to $50 \%$ relaxation (msec) & $101.2 \pm 3.3$ & $157.9 \pm 13.0^{*}<0.0001$ & $130.3 \pm 9.6^{*} 0.0039$ \\
\hline Number of cells & 70 & 31 & 39 \\
\hline
\end{tabular}

Values are presented as mean \pm standard error of the mean. Nomothermia, Untreated myocytes maintained at $37^{\circ} \mathrm{C}$ in media; Cardioplegia, incubated with Krebs buffer containing ethanol $(0.01 \%)$ followed by incubation with crystalloid cardioplegia ( $\left.\mathrm{K}^{+}, 24 \mathrm{mEq} / \mathrm{L}\right)$ at $4^{\circ} \mathrm{C}$ for 2 hours followed by rewarming with media at $37^{\circ} \mathrm{C}$ for 5 minutes; PMA/cardioplegia, pretreatment with $10^{-9} \mathrm{~mol} / \mathrm{L}$ of phorbol 12 -myristate 13 -acetate for 3 minutes followed by cardioplegic arrest with rewarming.

$* p<0.05$ versus normothermia. Values following the asterisk indicate specific $p$ values for each comparison.

subsequent reperfusion and rewarming. Specifically, myocyte percent shortening, shortening velocity, and velocity of relengthening were significantly increased from cardioplegia-alone values. More important, these indices of contractile function with PMA pretreatment were similar to normothermic control values $(p>0.60)$. However, myocyte total duration of contraction, time to peak contraction, and time to $50 \%$ relaxation were prolonged with PMA pretreatment and cardioplegic arrest when compared with normothermic values. These results suggest that pretreatment with PMA before simulated cardioplegic arrest protected indices of myocyte contractile function with subsequent reperfusion and rewarming, but defects in the temporal aspects of myocyte contractile process persisted. Co-incubation with chelerythrine and PMA before cardioplegic arrest and rewarming abolished the protective effects of PMA pretreatment on steadystate myocyte shortening velocity (Fig. 3). In myocytes pretreated with chelerythrine before cardioplegic arrest $(n=21)$, myocyte percent and velocity of shortening were similar to cardioplegiaalone values $(2.5 \% \pm 0.2 \%, 26 \pm 3 \mu \mathrm{m} / \mathrm{sec}$, respectively). Therefore the protective effects on myocyte contractile function with PMA pretreatment before cardioplegic arrest and rewarming were likely mediated by specific activation of PKC.

To more carefully examine whether PMA pretreatment in this isolated myocyte preparation induced PKC activation, we performed intracellular localization for PKC. In normothermic control myocytes, intense staining for PKC could be localized to the cytosolic compartment. Specifically, a strong fluorescent signal corresponding to PKC was observed in the perinuclear region and surrounding the myofilament array (Fig. 4). In these untreated control myocytes, minimal diffuse staining could be observed along the sarcolemma. In PMA-treated myocytes, the fluorescent signal for PKC within the perinuclear space could still be detected; however, a strong punctate staining pattern was observed along the myocyte sarcolemma (Fig. 4). In all myocytes examined after PMA treatment, an intense fluorescent signal could be readily detected along the cytosolic/sarcolemmal interface. This altered fluorescent staining pattern in PMA-treated myocytes is consistent with PKC activation and translocation. ${ }^{21-24}$ In myocytes treated with both PMA and chelerythrine, the fluorescent staining for $\mathrm{PKC}$ was similar to that in normothermic control myocytes (Fig. 4). Specifically, strong cytosolic staining could be detected in perinuclear and myofilament compartments with minimal diffuse staining along the sarcolemma. These results provide further evidence to suggest that PMA treatment caused PKC activation in this isolated myocyte preparation.

\section{Discussion}

Cessation of myocardial contractile activity is commonly required for the conduct of many cardiac surgical procedures and is routinely achieved through the administration of a hypothermic, hyperkalemic cardioplegic solution. However, transient LV dysfunction can occur after cardioplegic arrest, which may persist in the early postoperative period. Transient, brief periods of myocardial ischemia have been demonstrated to confer a protective effect to the myocardium with subsequent prolonged periods of ischemia; this protective effect has been defined as the preconditioning phenomenon. ${ }^{1-3} \mathrm{~A}$ proposed intracellular mechanism for the protective effects of preconditioning is activation of PKC. . $^{70}$ Past studies have demonstrated that a brief interval of PKC activation before a prolonged period of ischemia provided protective effects similar to those 


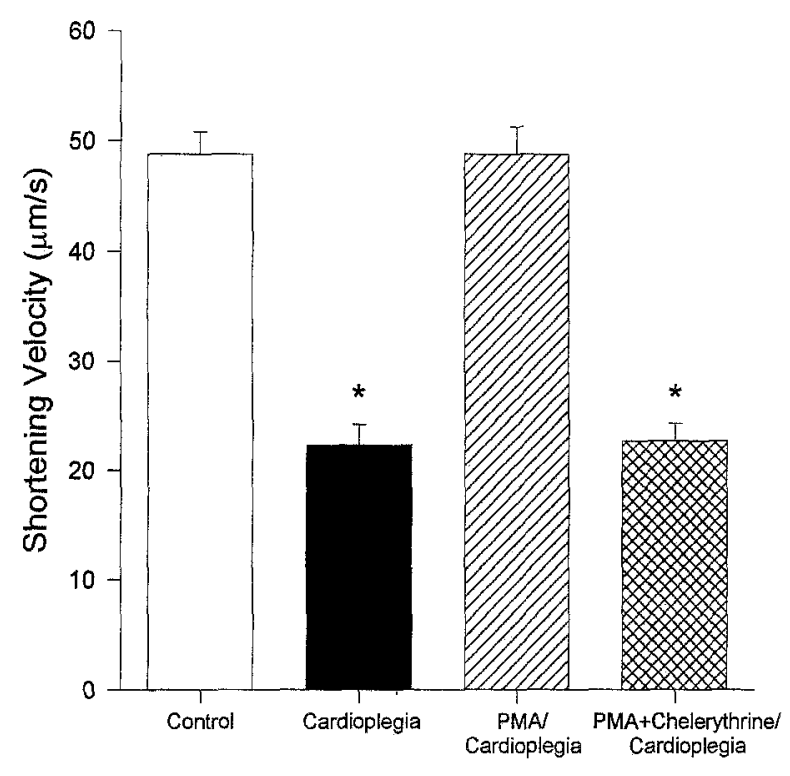

Fig. 3. A series of experiments was performed to determine more carefully whether the protective effect of pretreatment with PMA was due to specific activation of PKC. Accordingly, isolated myocytes were incubated in the presence of both chelerythrine $\left(10^{-6} \mathrm{~mol} / \mathrm{L}\right)$, a specific inhibitor of $\mathrm{PKC},{ }^{20}$ and PMA $\left(10^{-9} \mathrm{~mol} / \mathrm{L}\right)$ for 3 minutes and then subjected to cardioplegic arrest and rewarming. Two hours of simulated cardioplegic arrest and rewarming reduced steady-state myocyte shortening velocity compared with normothermic values. Pretreatment with PMA $\left(10^{-9} \mathrm{~mol} / \mathrm{L}\right)$ before cardioplegic arrest and rewarming restored steadystate myocyte shortening velocity to normothermic values. The protective effects of PMA pretreatment were abolished with co-incubation with chelerythrine and PMA. Thus the protective effects of PMA pretreatment were likely due to specific activation of PKC. ${ }^{*} p<0.05$ versus normothermia.

achieved by ischemic preconditioning. ${ }^{7-10}$ The present study was designed to determine whether a brief interval of PKC activation before prolonged cardioplegic arrest would provide protective effects on myocyte contractile function with subsequent reperfusion and rewarming. Using an isolated myocyte model of simulated cardioplegic arrest, we made two important observations. First, a brief period of PKC activation achieved through exposure of myocytes to the phorbol ester PMA before cardioplegic arrest provided protective effects on myocyte contractile function with rewarming. Second, the protective effects of PMA pretreatment before cardioplegic arrest and rewarming were abolished by concomitant treatment with a specific inhibitor of PKC. Therefore the present study provides direct evidence that a brief period of PKC activation
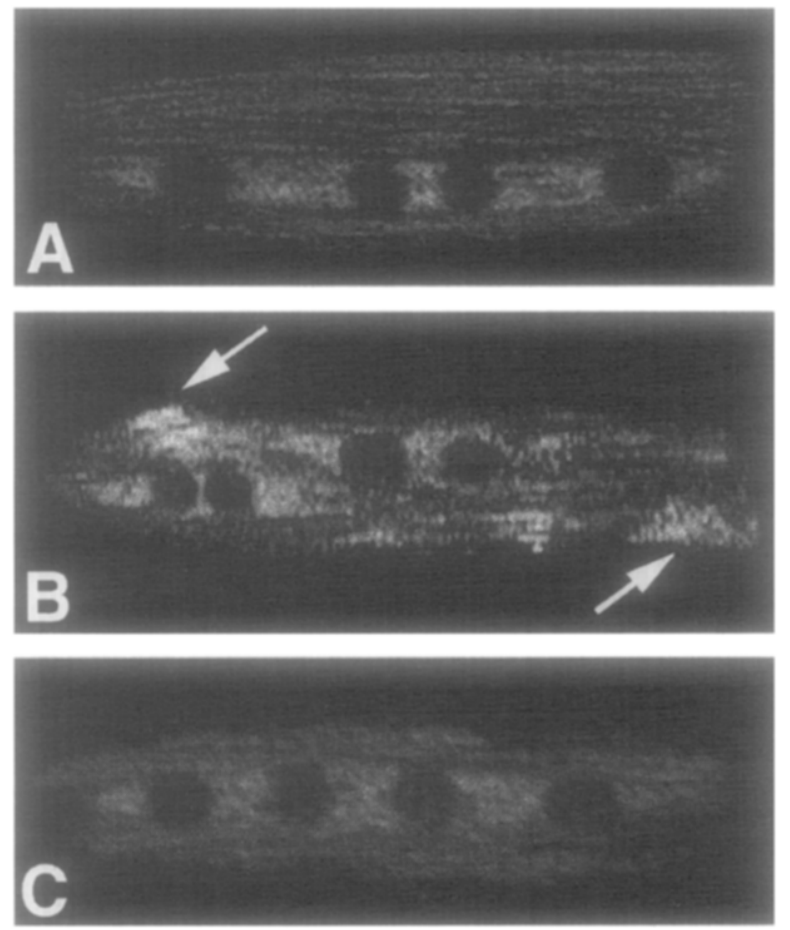

Fig. 4. To examine more carefully whether PMA pretreatment in this isolated myocyte preparation induced PKC activation, we used a fluorescent probe to perform intracellular localization. A, In control myocytes, intense staining for PKC could be localized to the cytosolic compartment. A strong fluorescent signal corresponding to PKC was observed in the perinuclear region and surrounding the myofilament array. B, In PMA-treated myocytes, the fluorescent signal for PKC within the perinuclear space could still be detected, but a strong punctate staining pattern was observed along the myocyte sarcolemma (arrows). C, In myocytes treated with both PMA and chelerythrine, the fluorescent staining for PKC was similar to normothermic control myocytes. These results provided evidence to suggest that PMA treatment caused PKC activation and subsequent translocation in this isolated myocyte preparation. All images were obtained with the use of scanning confocal microscopy with an image depth of $15 \mu \mathrm{m}$ and an original magnification of $600 \times$.

within the LV myocyte before cardioplegic arrest confers protective effects on myocyte contractile processes with subsequent reperfusion and rewarming.

In a past report, Illes and associates ${ }^{4}$ demonstrated that preconditioning in the rabbit heart improved LV pump function after hypothermic cardioplegic arrest and rewarming. A number of recent studies have provided evidence that an intracellular 
event necessary for inducing the preconditioning phenomenon is through activation of PKC. ${ }^{7-10,12,13}$ Past studies have demonstrated that preconditioning can be successfully elicited in isolated myocyte systems. ${ }^{12,13,16-19}$ In a recent report from this laboratory, it was demonstrated that hypoxic preconditioning of isolated myocytes before simulated cardioplegic arrest improved contractile function with subsequent reperfusion and rewarming. ${ }^{16}$ However, it remained unclear whether PKC activation before cardioplegic arrest would provide similar protective effects with respect to myocyte contractile function. In the present study, treatment with the phorbol ester PMA before prolonged cardioplegic arrest, significantly improved indices of myocyte contractile function with subsequent reperfusion and rewarming. Treatment of this isolated myocyte preparation with PMA caused a detectable intracellular shift in PKC from the cytoplasm to the sarcolemmal surface, consistent with PKC activation. ${ }^{21-24}$ Furthermore, co-treatment with a PKC inhibitor and PMA eliminated the protective effects induced by PMA pretreatment. Taken together, the results from the present study build on past reports and provide direct evidence that induction of PKC activation in isolated myocytes immediately before cardioplegic arrest will result in improved contractile function with subsequent reperfusion and rewarming.

Consistent with past reports, ${ }^{25-28}$ simulated cardioplegic arrest in an isolated LV myocyte system caused a significant reduction in the indices of myocyte contractile performance with subsequent reperfusion and rewarming. In the present study, a brief (3-minute) interval of PKC activation before the induction of cardioplegic arrest normalized myocyte percent and velocity of shortening with subsequent reperfusion and rewarming. This observation suggests that activation of PKC within the myocyte provided protective effects on myocyte homeostatic processes during the period of cardioplegic arrest. Activation of PKC results in a number of intracellular events within the myocyte, which include influencing ion exchange systems and pumps, activity of mitogen-activated protein kinases, phosphorylation states of contractile proteins, and cytoskeletal protein conformation. ${ }^{21,22,25,26}$ The activation of PKC has also been shown to influence gene expression and myocardial growth properties. ${ }^{21,22}$ Results from the present study, as well as past reports, ${ }^{7-10}$ demonstrated significant protective effects after only a brief period of PKC activation. Therefore the acute effects of PKC activation that were observed in the present study were probably not due to changes in transcriptional or translational processes, but more likely due to alterations in intracellular ionic homeostasis and phosphorylation states. PKC activational status has been demonstrated to influence the activity of the $\mathrm{Na}^{+} / \mathrm{H}^{+}$ exchanger, which will cause intracellular alkalinization. $^{21,22}$ PKC activation has also been demonstrated to influence intracellular $\mathrm{Ca}^{+2}$ levels by modulating the function of the 1-type $\mathrm{Ca}^{+2}$ channel on the myocyte sarcolemma, ${ }^{26}$ as well as the sarcoplasmic reticulum $\mathrm{Ca}^{+2}$-adenotriphosphatase. ${ }^{21}$ Thus, in the present study, it is likely that PKC activation in isolated $\mathrm{LV}$ myocytes changed intracellular $\mathrm{pH}$ and $\mathrm{Ca}^{+2}$ levels, which provided a protective effect when subjected to prolonged hypothermic, hyperkalemic cardioplegic arrest. However, future studies that directly measure intracellular $\mathrm{pH}$ and $\mathrm{Ca}^{+2}$ with PKC activation during cardioplegic arrest will be necessary to directly examine this possibility.

Pretreatment of isolated myocytes with the phorbol ester PMA, and therefore activation of PKC, before cardioplegic arrest improved myocyte shortening and velocity of shortening with subsequent reperfusion and rewarming. However, abnormalities in temporal indices of the contraction process, such as time to peak contraction, duration of contraction, and time to $50 \%$ relaxation, were prolonged with PMA pretreatment when compared with normothermic values. Myocyte percent shortening reflects the maximal extent of myofilament interaction and velocity of shortening reflects the rate of crossbridge cycling. PMA pretreatment increased the time interval to peak myocyte contraction, but myocyte percent shortening was similar to normothermic values. These findings suggest that $\mathrm{PKC}$ activation before cardioplegic arrest likely influenced mechanisms by which cross-bridge cycling rates are translated into overall myocyte shortening. It has been demonstrated previously that PKC activation influences the phosphorylation state and orientation of a number of contractile and cytoskeletal proteins. ${ }^{21,22,25}$ Thus, in the present study, PKC pretreatment may have altered cytoskeletal architecture within the myocyte, which in turn would influence sarcomere shortening to be translated to cell shortening. The present study demonstrated that an index of myocyte active relaxation, the time to $50 \%$ relaxation, remained prolonged with PMA pretreatment. The time to $50 \%$ relaxation reflects the period of cross-bridge release and $\mathrm{Ca}^{+2}$ resequestration by the sarcoplasmic reticu- 
lum. The findings of the present study suggest that the time necessary for cross-bridge release and the return of the myofilament array to a resting position was altered after cardioplegic arrest and could not be reversed by PMA pretreatment. PKC activation influences a number of $\mathrm{Ca}^{+2}$ homeostatic mechanisms within the myocyte. ${ }^{21,22,25,26}$ However, whether the persistent abnormalities in active relaxation with PMA pretreatment followed by cardioplegic arrest that were observed in the present study were due to temporal changes in myocyte $\mathrm{Ca}^{+2}$ homeostatic properties remains unclear. In light of the findings from the present study and the spectrum of intracellular events that occur after PKC activation, future studies that more carefully examine $\mathrm{Ca}^{+2}$ homeostatic processes with $\mathrm{PKC}$ activation and cardioplegic arrest are warranted.

In the present study, PKC activation under normothermic steady-state conditions reduced indices of myocyte contractile function. Karmazyn, Watson, and Moffat ${ }^{27}$ demonstrated that PMA treatment in rat heart preparations reduced indices of LV contractile performance. In studies of ischemic preconditioning in which a period of myocardial protection is conferred, the interval before the prolonged ischemic insult is often associated with a condition described as "myocardial stunning." ${ }^{1-3}$ Several past reports have suggested that a contributory intracellular mechanism for this "myocardial stunning" phenomenon is PKC activation. ${ }^{7-13}$ Thus the reduction in myocyte contractile performance that was observed in the present study after the acute administration of the phorbol ester PMA, with subsequent PKC activation, was not unexpected. In fact, the dose of PMA that caused a decline in steady-state function was used as a criterion that significant PKC activation had occurred within the myocyte. ${ }^{11,19,27}$ However, it may be possible to develop a protocol by which adequate PKC activation can be achieved to confer myocardial protection without a concomitant fall in contractile performance. Future studies with the myocyte model of simulated cardioplegic arrest described in the present study and in past reports, ${ }^{14-16}$ but which more carefully address this issue, would be appropriate.

Intracellular activation of PKC in most cell types is normally through the production of diacylglycerol, which is produced by a number of receptor-mediated events. 12, 21, 22, 26, 28, 29 Tumor-promoting phorbol esters such as PMA are membrane permeable and have structures similar to diacylglycerol, which provide a means to activate PKC directly. ${ }^{21,22,29}$ In addition to phorbol esters being described as tumorpromoting agents, these compounds are not effciently degraded by intrinsic enzymatic pathways and can cause a number of systemic effects. ${ }^{29}$ Thus the clinical application of phorbol esters such as PMA to induce PKC activation within the LV myocardium is problematic. However, several sarcolemmal receptor transduction systems have been demonstrated to induced PKC activation..$^{21,22}$ For , example, activation of the adenosine receptor system(s) that are located within the myocyte sarcolemma cause activation of PKC. ${ }^{3,12}$ Furthermore, Armstrong, Downey, and Ganote ${ }^{12}$ demonstrated that the protective effects of adenosine-receptor activation in the setting of global ischemia could be abolished through inhibition of PKC activation. ${ }^{12}$ Future studies that directly determine the relationship between the adenosine receptor and PKC activational states in the setting of cardioplegic arrest are warranted.

The isolated myocyte model used in the present study provides an uncomplicated means by which to determine the direct effects of pretreatment strategies with simulated cardioplegic arrest on contractile performance. Although this myocyte system has some advantages over an in vivo preparation, it has important limitations that must be recognized. The isolated myocyte system removes differences in loading conditions, neurohormonal activity, and the influence of nonmyocyte cell populations that would be encountered in vivo after cardioplegic arrest and rewarming. Another important consideration is that this isolated myocyte system differs from in vivo preparations in which capillary diffusion distances are affected by coronary artery disease, hypertrophy, and nonuniform maintenance and control of temperature. The limitations of the isolated myocyte system must be recognized, and extrapolation of the results from these in vitro studies to in vivo conditions should be performed with caution. In the present study, a qualitative morphometric examination was performed to determine the relative PKC activational state after PMA treatment. Although this portion of the study demonstrated that PMA treatment was associated with PKC translocation and that this brief interval of PKC activation before cardioplegic arrest improved myocyte function with subsequent rewarming, quantitation of the degree and nature of PKC activation within the myocytes was not addressed. PKC is not a single entity but comprises a large family of kinases encoded by different genes. ${ }^{21,22}$ PKC has been broadly categorized into three separate classes, based primarily on 
$\mathrm{Ca}^{+2}$ sensitivity of the amino-terminal regulatory region. ${ }^{22}$ At present, more than 10 isoforms of PKC have been identified, and the relative expression of these isoforms within the myocardium is an area of active investigation. ${ }^{21}$ Therefore, on the basis of the findings of the present study, future studies that more carefully examine the specific isoforms of PKC which can be recruited to provide protective effects in the setting of cardioplegic arrest would be appropriate. Nevertheless, the results from this study demonstrated that an endogenous means of providing improved myocardial protection during prolonged hypothermic, hyperkalemic cardioplegic arrest can be achieved through a brief period of PKC activation.

We appreciate the assistance of Latha Hebbar and Melissa Doscher in the execution of this project.

\section{REFERENCES}

1. Murry CE, Jennings RB, Reimer KA. Preconditioning with ischemia: a delay of lethal cell injury in ischemic myocardium. Circulation 1986;74:1124-35.

2. Przyklenk K, Kloner RA, Yellon DM. Ischemic preconditioning: the concept of endogenous cardioprotection. Przyklenk $\mathrm{K}$, Kloner RA, Yellon DM, editors. Boston: Kluwer Academic Publishers; 1994. p. 153-70.

3. Liu GS, Thornton JD, Van Winkle DM, Stanley AWH, Olsson RA, Downey JM. Protection against infarction afforded by preconditioning is mediated by $\mathrm{A}_{1}$-adenosine receptors in rabbit heart. Circulation 1991;84:350-6.

4. Illes RW, Wright JK, Inners-McBride K, Yang CJ, Tristan A. Ischemic preconditioning improves preservation with crystalloid cardioplegia. Ann Thorac Surg 1994;58:1481-5.

5. Bolling SF, Olszanski DA, Childs KF, Gallagher KP, Ning $\mathrm{XH}$. Stunning, preconditioning and functional recovery after global myocardial ischemia. Ann Thorac Surg 1994;58:822-7.

6. Galinanes M, Argano V, Hearse DJ. Can ischemic preconditioning ensure optinal myocardial protection when delivery of cardioplegia is impaired? Circulation 1995:92(Suppl); III389-4.

7. Speechly-Dick ME, Grover GJ, Yellon DM. Does ischemic preconditioning in the human involve protein kinase $\mathrm{C}$ and the ATP-dependent $\mathrm{K}^{+}$channel? Circ Res 1995;77:1030-5.

8. Bugge $\mathrm{E}$, Ytrehus $\mathrm{K}$. Ischaemic preconditioning is protein kinase $\mathrm{C}$ dependent but not through stimulation of $\alpha$-adrenergic or adenosine receptors in the isolated rat heart. Cardiovasc Res 1995;29:401-6.

9. Mitchell MB, Meng X, Ao L, Brown JM, Harken AH, Banerjee A. Preconditioning of isolated rat heart is mediated by protein kinase C. Circ Res 1995;76:73-81.

10. Cave AC, Apstein CS. Polymyxin B, a protein kinase C inhibitor, abolishes preconditioning-induced protection against contractile dysfunction in isolated blood perfused rat heart. J Mol Cell Cardiol 1996;28:977-87.

11. Capogrossi MC, Kaku T, Filburn CR, Pelto DJ, Hansford RG, Spurgeon HA, et al. Phorbol esters and dioctanoylglycerol stimulate membrane association of protein kinase $\mathrm{C}$ and have a negative inotropic effect mediated by changes in cytosolic $\mathrm{Ca}^{2+}$ in adult rat cardiac myocytes. Circ Res 1990;66:1143-55.
12. Armstrong S, Downey JM, Ganote CE. Preconditioning of isolated rabbit cardiomyocytes: induction by metabolic stress and blockade by the adenosine antagonist SPT and calphos: tin C, a protein kinase C inhibitor. Cardiovasc Res 1994;28: $72-7$.

13. Armstrong $\mathrm{S}$, Ganote $\mathrm{CE}$. Preconditioning of isolated rabbit cardiomyocytes: effects of glycolytic blockade, phorbol esters, and ischaemia. Cardiovasc Res 1994;28:1700-6.

14. Handy JR, Spinale FG, Mukherjee R, Crawford FA. Hypothermic potassium cardioplegia impairs myocyte recovery of contractility and inotropy. J Thorac Cardiovase Surg 1994; 107:1050-8.

15. Cavallo MJ, Dorman BH, Spinale FG, Roy RC. Myocyte contractile responsiveness after hypothermic, hyperkalemic cardioplegic arrest: disparity between exogenous calcium and $\beta$-adrenergic stimulation. Anesthesiology 1995;82:926-39.

16. Zellner JL, Hebbar L, Crawford FA, Mukherjee R, Spinale FG. Preconditioning has direct and beneficial effects on myocyte contractile processes with cardioplegic arrest and rewarming. Ann Thorac Surg 1996;61:558-64.

17. Ikonomidis JS, Tumiati LC, Weisel RD, Mickle DAG, Li RK. Preconditioning human ventricular cardiomyocytes with brief periods of simulated ischaemia. Cardiovasc Res 1994; 28:1285-91.

18. Armstrong S, Liu GS, Downey JM, Ganote CE. Potassium channels and preconditioning of isolated rabbit cardiomyocytes: effects of glyburide and pinacidil. J Mol Cell Cardiol 1995;27:1765-74.

19. Johnson JA, Mochly-Rosen D. Inhibition of the spontaneous rate of contraction of neonatal cardiac myocytes by protein kinase C isozymes: a putative role for the $\epsilon$ isozyme. Circ Res 1995;76:654-63.

20. Herbert JM, Augereau JM, Gieye J, Maffrand JP. Chelerythrine is a potent and specific inhibitor of protein kinase $\mathrm{C}$. Biochem Biophys Res Commun 1990;172:993-9.

21. Sugden $\mathrm{PH}$, Bogoyevitch MA. Intracellular signalling through protein kinases in the heart. Cardiovasc Res 1995; 30:478-92.

22. Steinberg SF, Goldberg M, Rybin VO. Protein kinase C isoform diversity in the heart. J Mol Cell Cardiol 1995;27; 141-53.

23. Disatnik MH, Buraggi G, Mochly-Rosen D. Localization of protein kinase $\mathrm{C}$ isozymes in cardiac myocytes. Exp Cell Res 1994:210:287-97.

24. Chen CS, Poenie M. New fluorescent probes for protein kinase C: synthesis, characterization and application. J Biol Chem 1993;268:15812-2.

25. Clark EA, Brugge JS. Integrins and signal transduction pathways: the road taken. Science 1995;268:233-9.

26. Dösemeci A, Dhallan RS, Cohen NM, Lederer WJ, Rogers TB. Phorbol ester increases calcium current and simulates the effects of angiotensin II on cultured neonatal rat heart myocytes. Circ Res 1988;62:347-57.

27. Karmazyn M, Watson JE, Moffat MP. Mechanisms for cardiac depression induced by phorbol myristate acetate in working rat hearts. Br J Pharmacol 1990;100:826-30.

28. MacLeod KT, Harding SE. Effects of phorbol ester on contraction, intracellular $\mathrm{pH}$ and intracellular $\mathrm{Ca}^{2+}$ in isolated mammalian ventricular myocytes. J Physiol 1991;444: 481-98.

29. Nishizuka Y. Studies and perspectives of protein kinase C. Science 1986;233;305-12. 